\title{
Valoración del procesamiento ortográfico en niños españoles con dislexia: El papel de las unidades léxicas y subléxicas
}

\author{
Mercedes Rodrigo ${ }^{1,}$ Juan E. Jiménez ${ }^{1}$, Eduardo \\ García $^{1}$, Alicia Díaz ${ }^{1}, M^{a}$ Rosario Ortiz ${ }^{1}$, Remedios \\ Guzmán $^{3}$, Isabel Hernández-Valle ${ }^{1}$, Adelina Estévez ${ }^{2}$, \\ y Sergio Hernández ${ }^{4}$
}

\footnotetext{
${ }^{1}$ Dpto. de Psicología Evolutiva y de la Educación.

${ }^{2}$ Dpto. de Psicología Cognitiva Social y Organizacional.

${ }^{3}$ Dpto. de Didáctica e Investigación Educativa y del Comportamiento, ${ }^{4}$ Dpto. de Psicobiología y Metodología de las Ciencias del Comportamiento. Universidad de La Laguna, Tenerife .
}

España

mrodrigo@ull.es

Esta investigación ha sido financiada por los Fondos Europeos para el Desarrollo Regional (FEDER), 1FD97-1140, y Dirección General de Investigación Científica y Técnica (DIGICYT). 


\section{Resumen}

Introducción. El objetivo de este estudio ha sido examinar el papel de las unidades multiletras, tales como el morfema y la palabra completa, en el acceso al léxico, en niños españoles con dislexia.

Método. Para llevarlo a cabo, se seleccionó una muestra la cual se distribuyó en tres grupos diferentes: 1) Un grupo experimental formado por 18 niños con dificultad lectora (DAL); (2) Un grupo control de 27 lectores normales de la misma edad que el grupo de lectores con dificultad (BL); y (3) Un grupo control formado por 15 niños más jóvenes y del mismo nivel lector que los lectores con dificultad (NL). Se utilizó una tarea de selección de homófonos y de comprensión de raíces morfológicas. Se analizaron los resultados teniendo en cuenta medidas de exactitud y de latencia para la tarea de comprensión morfológica y de exactitud para la tarea de homófonos.

Resultados. Los resultados mostraron que había un déficit en el procesamiento léxico en los niños con dificultad lectora ya que su rendimiento era más bajo que el de los niños más jóvenes con el mismo nivel lector. Sin embargo, cuando analizamos la latencia de la tarea de comprensión morfológica, los resultados mostraron que esta latencia disminuía, independientemente de los grupos, a medida que avanzaban las presentaciones, aunque el rendimiento del grupo de lectores normales, emparejados en edad con los lectores con dificultad, era significativamente mejor que el de los otros dos grupos.

Palabras clave: Dificultad lectora, dislexia, procesamiento léxico, procesamiento morfológico. 


\section{Introducción}

El reconocimiento de la palabra es un paso importante en el desarrollo de la lectura y constituye uno de los principales déficits en los niños con dificultad lectora. (Perrfetti, 1986, 1989; Siegel, 1986). Numerosos estudios sugieren que los niños con dificultad lectora (DAL) muestran déficits en los procesos léxicos y subléxicos (e.g., Beech y Awaida, 1992; Ehri y Wilce, 1983; Manis, 1985; Perfetti, 1985). Otras investigaciones han demostrado que los estudiantes con DAL son más lentos en el acceso al léxico que los lectores competentes (e.g., Cirrin, 1984; Ellis, 1981; Johnston y Thompsom, 1989; Laxon, Coltheart y Keaping, 1988; Rayner, 1988; Seymour, 1987; Seymour y Porpodas, 1980).

La lengua española es un sistema alfabético transparente (la ortografía está ensamblada sobre la estructura fonológica), en la que las reglas de conversión letra-sonido son bastantes sencillas y tienen pocas excepciones ya que hay una correspondencia directa entre el símbolo escrito y el fonema.. Varios estudios han puesto de manifiesto que, en español, la lectura se lleva a cabo a través del procesamiento fonológico (De Vega, Carreiras, Gutiérrez Calvo y Alonso Quecuty, 1990). Sin embargo, el procesamiento léxico tiene un valor funcional en el reconocimiento de la palabra en una lengua transparente ya que varios estudios con adultos españoles (e.g., Domínguez y Cuetos, 1992; Domínguez, Cuetos y De Vega, 1993) y niños (Jiménez y Rodrigo, 1994; Rodrigo y Jiménez, 1999; Rodrigo y Jiménez, 2000; Valle, 1989), han mostrado que variables como la lexicalidad y la familiaridad de las palabras tienen una efecto similar al que se produce en un sistema ortográfico opaco..Esto significa que los lectores españoles utilizan la ruta léxica u ortográfica para identificar las palabras familiares.

La mayoría de los estudios proponen que los problemas en el reconocimiento de la palabra que muestran los lectores con dificultad están básicamente en la ruta fonológica (Rack, Snowling y Olson, 1992; Siegel y Ryan, 1988; Stanovich, 1988; Wagner y Torgesen, 1987). Treiman (1992), ha sugerido que la correspondencia entre la letra impresa y el habla podría no estar restringida al nivel de la palabra completa o al nivel de fonema. Ella puntualizó que unidades multiletras como el onset o la rima podrían ser más importantes que otros tipos de unidades en el procesamiento de las palabras impresas, en niños y en adultos lectores. La representación impresa del habla podría ser más fácilmente comprendida, 
especialmente por los niños con pobres habilidades fonológicas, si fuera accedida a través de unidades más grandes que el fonema. Álvarez, Carreiras y Taft (2001) estudiaron, en participantes adultos españoles, la utilización en el reconocimiento de la palabra de tres tipos de unidades multiletras: Estructura silábica básica ortográfica (BOSS), el morfema raíz y la sílaba. Se encontró un nulo efecto del BOSS, excepto cuando tenía la misma frecuencia que el morfema raíz. Estudios previos confirman que el BOSS no es una unidad funcional en español (Sánchez-Casas, 1996). Con relación a la unidad morfológica, la frecuencia de la raíz produce un efecto facilitatorio en el reconocimiento de la palabra. Asimismo, hay considerable evidencia de que la sílaba es una importante unidad de procesamiento en español. Cuando sílabas de alta frecuencia, en primera posición, forman la palabra, dificultan su reconocimiento, siendo más rápido el reconocimiento de palabras formadas pos sílabas de baja frecuencia en la primera posición. Estos resultados son consistentes con aquellos estudios previos en español que mostraron que la frecuencia silábica en primera posición de la palabra produce un efecto inhibitorio en lectores competentes (Álvarez, de Vega y Carreiras, 1998; Jiménez y Rodrigo, 1994; Rodrigo y Jiménez, 2000). Sin embargo, se encontraron efectos facilitatorios de la frecuencia silábica en niños que estaban aprendiendo a leer (Jiménez, Guzmán y Artiles, 1997).

Desde la propuesta de Treiman, se ha llevado a cabo multitud de investigaciones sobre el papel de las unidades multiletras en el reconocimiento de la palabra en niños con dificultad lectora.. En español, Jiménez (1997), analizó la conciencia fonémica en un diseño de nivel lector y los resultados demostraron que no había diferencias en la conciencia intrasilábica entre el rendimiento de los lectores con dificultad y el grupo control de lectores más jóvenes emparejados por el nivel lector, pero había diferencias en la ejecución de la tarea fonémica (e.g., segmentación y síntesis de fonemas) porque el grupo DAL rendía más pobremente que el grupo control de niños más jóvenes.. Esta diferencia sugiere que un precursor de la dificultad en la codificación fonológica podría ser un déficit en las habilidades de segmentación de la lengua. Más recientemente, Jiménez, Álvarez, Estévez y Hernández-Valle (2000), indicaron que no había diferencias entre niños españoles con dificultad lectora y lectores normales en la utilización de correspondencias que están basadas en unidades más grandes que el fonema, como el onset y la rima. Por consiguiente, como Bryant y Goswami (1986) han sugerido, si no se encuentran diferencias entre grupos con DAL y los grupos control, emparejados por la edad y por el nivel lector, a pesar del hecho de que el grupo con dificultad lectora tiene el nivel mucho más bajo, entonces es poco probable que la variable en 
cuestión juegue algún papel en causar la dificultad lectora. Estos resultados sugieren que las unidades onset y rima no son relevantes en el reconocimiento de la palabra en lengua española porque hay una correspondencia directa entre el grafema y el fonema.

Con relación a la sílaba, otra unidad multiletras que podría intervenir en el acceso al léxico, Jiménez y Rodrigo (1994) encontraron que no había interacciones entre la frecuencia silábica en las palabras y el nivel lector. Esto implica que el fallo del grupo con dificultad lectora se encuentra en la conversión grafema fonema y no a nivel de sílaba. Estos resultados empíricos sugieren que es bastante improbable que la unidad de la sílaba juegue algún papel en la explicación de la causa de la dificultad lectora.

Otra unidad multiletras en el acceso al léxico es el morfema. El morfema es la unidad más pequeña de la lengua escrita con significado. Las palabras están morfológicamente articuladas y estructuradas. La investigación sobre el procesamiento morfológico empieza con el informe de Taft y Forster (1976) en el que se propone que los prefijos de las palabras son analizados, dentro de su constituyente, antes de que el acceso al léxico ocurra. El español es una lengua con un sistema inflexional muy complejo, con sufijos de género y número para los nombres y los adjetivos y un alto grado inflexional en los verbos. Sin embargo hay pocos estudios sobre el papel del morfema en el reconocimiento de las palabras. Domínguez, Cuetos y Seguí (2000) estudiaron el acceso al léxico para investigar si los significados de las palabras se obtenían indirectamente a través de sus morfemas (full parsing) o, por el contrario, se obtenían por un procedimiento directo, semejante al que se utiliza cuando buscamos en un diccionario (full listing), esto es, accediendo a su significado en la memoria. Estos autores concluyeron que la mejor opción para las palabras morfológicamente irregulares podría ser almacenar todas las formas, separadamente, en el lexicón.. Por el contrario, las palabras morfológicamente regulares podrían ser reconocidas después de analizar los sufijos por reglas. De la misma manera, Domínguez, Seguí y Cuetos (2002) han intentado disociar las fuentes de procesamiento ortográfico, morfológico y semántico, mediante un paradigma de priming El efecto de facilitación obtenido por las palabras, morfológicamente relacionadas, en todos los experimentos, podría ser interpretado como una manera específica de acceso al léxico. Además, las palabras morfológicamente relacionadas (morfema raíz) no pueden ser consideradas como diferentes items léxicos con diferentes significados y distintas propiedades semánticas. Tampoco pueden ser candidatas competitivas entre sí (Alvarez, Carreiras y Taft, 
2001). La mayoría de estos estudios han sido llevados a cabo con adultos españoles, no con niños con dificultades lectoras.

Sin embargo, hay estudios con niños como los de Tornéus (1987) que encontraron que la conciencia morfológica de los niños de kindergarten predecía su habilidad lectora en el segundo grado. En otro estudio sobre la estrategia lectora de disléxicos y lectores más jóvenes del grupo control, emparejados por el nivel lector, Elbro y Petersen (1993) encontraron que los adolescentes con dislexia leían prácticamente tan bien en la condición de morfema como en la de palabra completa y estos participantes se apoyaban más en la condición de morfema que los lectores normales más jóvenes. Los resultados de los estudios de Elbro and Arnbak, (1996) muestran que los adolescentes con dislexia utilizan el reconocimiento del morfema raíz como una estrategia compensatoria en la lectura de palabras aisladas y de texto coherente. Los mismos autores sugieren que es posible mejorar la conciencia morfológica independientemente de la conciencia fonémica y tal entrenamiento puede tener efectos positivos sobre la lectura coherente de un texto, la exactitud del deletreo o las palabras morfológicamente complejas. Todas estas evidencias apoyan el punto de vista de que el entrenamiento en el reconocimiento del morfema podría funcionar como una estrategia compensatoria para los lectores con problemas. Unas buenas habilidades morfológicas podrían ayudar a los lectores que tienen problemas fonológicos para ser más fluidos (Elbro y Arnbak, 1996).Sin embargo, estos estudios no han sido llevados a cabo en una lengua transparente como el español, el cual es enteramente predecible sobre las bases de los fonemas, sino en ortografías que son gobernadas no solamente por la fonología sino también por la morfología. Por lo tanto, permanece la siguiente cuestión para ser contestada; ¿Contribuye el reconocimiento del morfema a la lectura de los niños con dislexia y es esta contribución diferente en los sujetos con dislexia y en los lectores normales en español?.

Finalmente, la otra unidad de acceso al léxico que hemos investigado en este trabajo es la palabra completa. La lectura en español se lleva a cabo a través del procesamiento fonológico porque es un sistema ortográfico transparente, el procesamiento léxico tiene un valor funcional en el reconocimiento de la palabra. Desde una perspectiva evolutiva, el modelo de etapas para aprender a leer, distingue entre una fase temprana de lectura, durante la que se aprende la correspondencia grafema-fonema (generalmente llamada etapa alfabética), y otra posterior durante la que la unidad de procesamiento es la palabra completa y en la que se adquiere las representaciones ortográficas o léxicas (la etapa ortográfica) (Frith, 1985; 
Seymour \& McGregor, 1984). Cuando los niños acceden a la etapa ortográfica, se puede decir que son expertos lectores porque son capaces de leer fluidamente. Los lectores normales, en su primera etapa de lectura podrían reconocer palabras frecuentes y desconocidas utilizando el sonido de la palabra. Posteriormente, estos lectores estar en condiciones de hacer uso de una estrategia léxica para reconocer las palabras familiares (Backman, Bruck, Herbert y Seidenberg, 1984). Coltheart (1987) especifica que el procesamiento fonológico precede al ortográfico cuando los niños están aprendiendo a leer. De la misma manera, Share y Stanovich (1995) encontraron que la decodificación fonológica facilita el establecimiento de las representaciones léxicas. Asimismo, Alegría (1985) ha sugerido que la construcción del lexicón depende de la ruta fonológica en español. Sin embargo, los niños con DAL tienen problemas en el procesamiento de las relaciones letra-sonido. Esta clase de problemas determina la pobre experiencia de estos niños con la lectura y, por consiguiente, tendrán menos representaciones léxica en su lexicón que los lectores normales de la misma edad (Stanovich, 1986). No obstante, Rodrigo y Jiménez (1999), analizando los errores en una tarea de nombrado encontraron evidencia de que los niños españoles con dificultad lectora utilizaban la estrategia ortográfica para compensar su déficit fonológico, pero su rendimiento en esta estrategia era más bajo que el de los buenos lectores.

Basado en estas evidencias empíricas, el objetivo de este estudio ha sido comprobar si el reconocimiento del morfema y de la palabra completa contribuye a la lectura en niños españoles con dislexia y si su contribución es diferente en niños con dificultad lectora y lectores normales. Se utilizó una tarea de comprensión morfológica y una tarea de selección de homófonos para valorar el procesamiento morfológico y léxico, respectivamente, de los sujetos. Nuestra predicción es que el grupo DAL podría tener más necesidad de utilizar unidades mayores como el morfema en el reconocimiento visual de la palabra, sin embargo, los grupos control podrían no necesitar unidades multiletras porque su conciencia fonémica puede ayudarles eficazmente en el reconocimiento de la palabra en una ortografía transparente como el español. Sin embargo, nosotros esperamos que el rendimiento de los niños con DAL podría ser más bajo que el de los otros grupos en la utilización de las estrategias léxicas porque tienen problemas en la utilización de estrategias fonológicas y el lexicón se construye sobre esta habilidad. 


\section{Método}

\section{Participantes}

Se seleccionó una muestra de 60 participantes (30 niños y 30 niñas) con un rango de edad de 7 a 12 años $(M=111.05 ; \mathrm{DT}=11.64)$. Los niños fueron clasificados en tres grupos: (1) Un grupo experimental de 18 niños con dificultad lectora (edad, M=117.16; DT=5.3), DAL; (2) Un grupo control de 27 lectores normales emparejados en edad con los niños con dificultad (edad, M= 117.11; DT= 5.0), BL; and (3) Un grupo control de 15 niños más jóvenes del mismo nivel lector que los lectores con dificultad (edad, M=92.8; DT=3.7), NL. Se definían niños con DAL aquellos que tenían una puntuación de percentil $<25$ sobre el test de Pseudopalabras. No había diferencias significativas en la distribución de los participantes en función del género $\mathrm{X}(2)=1.28, \mathrm{p}=.525$. ni diferencias entre grupos en el $\mathrm{CI}, \mathrm{F}(2,57)=.1,0 \mathrm{p}=.98$. Sin embargo, encontramos diferencias significativas entre los grupos en la memoria de trabajo verbal, $\mathrm{F}(2,58)=3.8, \mathrm{p}=.05$. Esto significa que los lectores con dificultad mostraban puntuaciones significativamente más bajas que los lectores normales emparejados por la edad $(\mathrm{t}=-.64$, $\mathrm{p}<.05)$ y que los lectores normales más jóvenes emparejados por el nivel lector $(\mathrm{t}=-.69$, $\mathrm{p}<.001$ ). Fueron excluidos de la muestra los niños que tenían déficits sensoriales, problemas neurológicos y otros problemas tradicionalmente utilizados como criterios de exclusión para las DAL. Los niños procedían de escuelas de área urbana y su procedencia socioeconómica era de nivel medio.

Tabla 1. Medias y desviaciones típicas de medidas de CI, edad y memoria de trabajo. $\mathrm{NL}=$ Nivel lector; $\mathrm{BL}=$ Buenos lectores.

\begin{tabular}{lcccccc} 
& \multicolumn{6}{c}{ Grupos } \\
\cline { 2 - 7 } & \multicolumn{2}{c}{ Dificultad lectora } & \multicolumn{2}{c}{ NL control } & \multicolumn{2}{c}{ BL control } \\
\cline { 2 - 7 } CI & $\mathbf{M}$ & $\mathbf{D T}$ & $\mathbf{M}$ & $\mathbf{D T}$ & M & DT \\
\cline { 2 - 7 } Edad & 109.8 & 13.7 & 110.3 & 13.1 & 109.8 & 13.3 \\
Memoria & 117.16 & 5.36 & 92.8 & 3.72 & 117.11 & 5.00 \\
de Trabajo & 2.61 & 0.6 & 3.26 & 0.9 & 3.06 & 0.7 \\
\hline
\end{tabular}




\section{Diseño}

Se llevaron a cabo dos diseños experimentales:

(1). Se llevó a cabo un diseño de nivel lector que consta de tres grupos (DAL, BL y NL) e intraparticipantes (tarea de comprensión morfológica y selección de homófonos). Los resultados fueron analizados utilizando respuestas correctas para la tarea de comprensión morfológica y para la tarea de homófonos. Se utilizó la medida de latencia para la tarea morfológica.

(2). Se utilizó el diseño de nivel lector que consta de tres grupos (DAL, BL y NL) e intraparticipantes (número de presentaciones, 1-4, en la tarea de comprensión morfológica). Nosotros analizamos como variables dependientes el tiempo de latencia de las respuestas correctas . Asimismo, controlamos el efecto de longitud dividiendo los tiempos de latencia de las respuestas correctas por el número de letras de cada palabra.

\section{Materiales}

Para seleccionar la muestra de este estudio se utilizó tres tests.

Test estandarizado de habilidades lectoras (PROLEC, Cuetos, Rodríguez, y Ruano, 1996). Esta prueba incluye diferentes subtest de lectura. Administramos solamente el subtest de lectura de Pseudopalabras porque la velocidad y la exactitud de nombrar pseudopalabras discrimina entre buenos lectores y lectores con dificultad en una ortografía opaca (Perfetti y Hogaboam, 1075; Siegel y Ryan, 1988; Siegel y Faux, 1989), y también en una ortografía transparente, como el español (Domínguez y Cuetos, 1992; Jiménez y Rodrigo, 1994). Este subtest requiere la identificación correcta de pesudopalabras, ya que mide la exactitud, con diferentes estructuras lingüísticas.

Tarea de Memoria de Trabajo Verbal. Para valorar la memoria de trabajo de los participantes se administró la tarea utilizada por Siegel y Ryan (1989). Esta tarea fue diseñada siguiendo el procedimiento desarrollado por Daneman y Carpenter (1980). Los niños escuchaban frases en las que se omitía la última palabra. La tarea consistía en completar la palabra que faltaba en cada frase y entonces repetir todas palabras perdidas del grupo de frases. Había tres pruebas en 
cada nivel o amplitud de grupo (2,3,4, y 5 palabras). Se paraba la prueba cuando el niño fallaba las tres pruebas de un nivel.

Test de Inteligencia libre de contenidos culturales (escala 1 y 2, Forma A, Cattell y Cattell, 1950/1989), Esta prueba nos permite medir el factor $g$ sin interferencias culturales.

Para valorar el procesamiento léxico y morfológico en nuestra muestra administramos las tareas morfológica y léxica que están incluidas en el SICOLE, un programa de computadora (Un sistema basado en el conocimiento para la evaluación de las dificultades lectoras en lengua española, Jiménez et al., 2002). SICOLE comprende diferentes componentes conectados serialmente. Se le presenta al usuario un menú que le lleva a seleccionar un grupo de situaciones de lenguaje y de lectura (e.g., percepción del habla, conciencia silábica, conciencia intrasilábica, conciencia fonémica, lectura de palabras, procesamiento de frases, procesamiento morfológico y léxico y comprensión lectora). La prueba morfológica y léxica incluye dos tareas: (1)Tarea de selección de homófonos (2) Tarea de comprensión de morfemas raíz.

En la tarea de selección de homófonos (homófonos son palabras que se deletrean de distinta manera, tienen la misma pronunciación y significan cosas distintas), se presenta a los participantes, con un dibujo, dos palabras homófonas y se les hace una pregunta (e.g. ¿cual es un animal?). Los niños tienen que elegir una de las palabras escritas. La respuesta correcta es la palabra que coincide con el dibujo y la pregunta. Se presentaban 9 items en la tarea de selección de homófonos (Fiabilidad: Alpha .97).

En la tarea de comprensión de morfema raíz, se presenta a los participantes una palabra escrita con dos dibujos. Uno de estos corresponde a la palabra escrita. El niño tiene que leer en voz alta las palabras escritas y señalar el dibujo correcto. Se utilizaron cinco diferentes morfemas raíz. Cada uno incluía la misma raíz durante las cuatro presentaciones, con el sufijo cambiados. Se administraron veinte items (Fiabilidad: Alpha.90).

\section{Procedimiento}

Seis psicólogos experimentados administraron el test de lectura y las pruebas de evaluación de la conciencia morfológica y léxica utilizando el software SICOLE. Ambas evaluaciones fueron llevadas a cabo individualmente y en cuatro sesiones, por participante, en un local del colegio del que procedía el niño y que reunía las condiciones apropiadas para este 
objetivo. Las tareas se presentaron al azar, cada una precedida de dos ejemplos para asegurase de que el participante había entendido las instrucciones..

\section{Resultados}

Procesamiento morfológico y léxico y dificultad lectora. La comparación entre niños con dificultad lectora y lectores normales emparejados por la edad y lectores normales más jóvenes fue llevada a cabo por el hecho de que el grupo de lectores con dificultad (DAL) presentaba un menor rendimiento en la memoria verbal de trabajo que los grupos control. Para controlar esta diferencia, se llevaron a cabo dos análisis de covarianza (ANCOVAs) a través de los grupos para las tareas de homófonos y morfema raíz en las que la memoria de trabajo verbal servía de covariante. La corrección de Bonferroni fue utilizada para cada una de estas ANCOVAs para reducir la posibilidad de cometer errores. Los resultados revelaron un efecto significativo de la MT en la tarea de selección de homófonos $[\mathrm{F}(1,59)=7.47, \mathrm{p}<.01]$. El mismo efecto significativo se encontró en la tarea de comprensión morfológica, exactitud, $[F(1,59)=18.77$, $\mathrm{p}<.001]$, y latencia $[\mathrm{F}(1,34)=3.59, \mathrm{p}<.001]$, lo que nos indica que la utilización del ANCOVA, es adecuado.

\section{Diferencias de Tareas}

A continuación se llevó a cabo un análisis de los datos obtenidos, utilizando un diseño factorial de $3 \times 2$, con un factor intergrupo de tres niveles (DAL; BL; y NL) y un factor intragrupo con dos tipos de tareas (selección de homófonos y comprensión morfológica). Se tomó como variable dependiente respuestas correctas y fue calculado, separadamente, a través de participantes (F1) y de items (F2). La Tabla 2 muestra las medias y las desviaciones típicas, para los tres grupos, en cada una de las tareas morfológica y léxica.

Tabla 2. Medias y desviaciones típicas de respuestas correctas en tareas ortográficas y de conciencia morfológica en función de los diferentes grupos.

$\mathrm{NL}=$ Nivel lector; $\mathrm{BL}=$ Buenos lectores.

\begin{tabular}{cccccc}
\hline \multicolumn{7}{c}{ Grupos } \\
\hline \multicolumn{2}{c}{ Dificultad lectora } & \multicolumn{2}{c}{ NL control } & \multicolumn{2}{c}{ BL control } \\
\hline M & DT & M & DT & M & DT \\
\hline .52 & .20 & .76 & .14 & .93 & .08 \\
.76 & .18 & .78 & .16 & .96 & .04 \\
\hline
\end{tabular}


Figura 1. Interacción entre grupos y diferentes tareas sobre respuestas correctas. $\mathrm{NL}=$ Nivel lector; $\mathbf{B L}=$ Buenos lectores; $\mathrm{DAL}=$ Dificultad lectora.

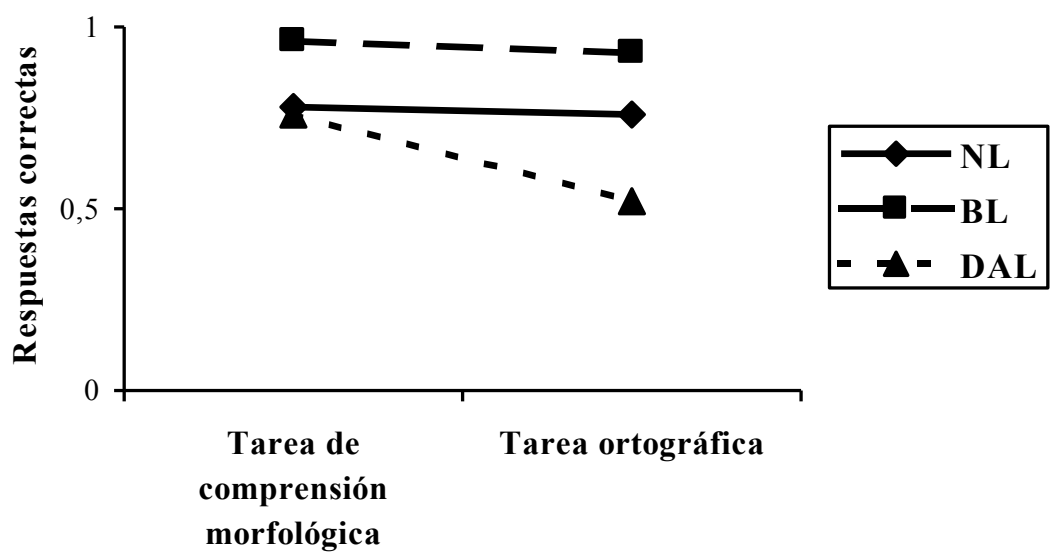

En el análisis aparece un efecto principal de Grupo $[\mathrm{F} 1(2,48)=36,72, \mathrm{p}<.001], \eta^{2}=.62$ [F2 $(2,34)=.23,002, \mathrm{p}<.001], \mathrm{y}$ también un efecto principal de Diferencia de Tarea $[\mathrm{F} 1(1,56)=$ $10.42 \mathrm{p}<.001], \eta^{2}=.11[\mathrm{~F} 2(1,34)=17.16, \mathrm{p}<.001]$, pero fue subsumido por la interacción significativa Grupo x Diferencia de Tarea $[\mathrm{F} 1(2,57)=3.39, \mathrm{p}<.01], \eta^{2}=.24[\mathrm{~F} 2(2,34)=.5$, $\mathrm{p}<.010]$. El efecto principal confirmó que el grupo de nivel lector (NL) puntuaba significativamente más alto que el DAL en la tarea de homófono $[F(1,48)=17.27, p<.001]$.Sin embargo, no había diferencias entre estos grupos en la tarea de comprensión morfológica.

Estos resultados indicaron que el grupo de BL tenía mejor rendimiento en las respuestas que el grupo NL en la tarea de homófonos $[F(1,48)=9.27, p<.001]$ y en la tarea de comprensión morfológica $[\mathrm{F}(1,48)=14,50, \mathrm{p}<.001]$. Las mismas diferencias significativas se encontraron entre el grupo de BL y NL en la tarea de homófonos $[\mathrm{F}(1,48)=71,33, \mathrm{p}<.001]$ y en la tarea morfológica $[\mathrm{F}(1,48)=19,48, \mathrm{p}<.001]$.

\section{$\underline{\text { Diferencias entre las tareas según los tiempos de latencia }}$}

Analizamos también las diferencias entre los grupos en los tiempos de latencia a lo largo de las distintas presentaciones de la tarea de comprensión morfológica (4 ítems o presentaciones consecutivas con un mismo morfema raíz). Llevamos a cabo un análisis de covarianza (ANCOVA) donde la latencia de los aciertos era la variable dependiente y se realizaron los análisis por sujetos e ítems. Los resultados indicaron un efecto principal de grupo $(\mathrm{F}(2,34)=3.53, \mathrm{p}<.01)$, y un efecto principal de momento de presentación $(\mathrm{F}(3,32)=51.52$, 
$\mathrm{p}<.001)$. Los lectores del grupo control EC fueron significativamente más rápidos que los lectores con dificultades $(\mathrm{F}(1,34)=5.34, \mathrm{p}<.05)$, y que los lectores más jóvenes del grupo NL $(\mathrm{F}(1,34)=4.34, \mathrm{p}<.05)$. Debido a la aparición de matrices singulares en los análisis de presentación recurrimos al análisis por pares de la prueba t, y corregimos su significación con Bonferroni. Al comparar los tiempos de latencia entre presentaciones observamos que existían diferencias significativas entre la primera y segunda presentación $\mathrm{t}=61.67, \mathrm{p}<.001$, también aparecieron diferencias entre la primera y tercera presentación $\mathrm{t}=9,26, \mathrm{p}<.001$, y entre la primera y cuarta $\mathrm{t}=94.10, \mathrm{p}<.001$. Finalmente, existían diferencias entre la segunda y cuarta presentación $\mathrm{t}=32.41, \mathrm{p}<.01$.

Tabla 3. Medias y desviaciones típicas de medidas de latencia/número de letras en tareas de comprensión morfológica a lo largo de presentaciones en función de los diferentes grupos. $\mathrm{NL}=$ Nivel lector; $\mathrm{BL}=$ Buenos lectores.

\begin{tabular}{ccccccc} 
& \multicolumn{7}{c}{ Grupos } \\
\cline { 2 - 7 } Presentaciones & \multicolumn{2}{c}{ Dificultad lectora } & \multicolumn{2}{c}{ NL control } & \multicolumn{2}{c}{ BL control } \\
\cline { 2 - 7 } & $\mathbf{M}$ & DT & M & DT & M & DT \\
\cline { 2 - 7 } $\mathbf{1}$ & 334.97 & 128.46 & 347.05 & 110.83 & 219.42 & 92.10 \\
$\mathbf{2}$ & 277.55 & 106.88 & 265.21 & 44.60 & 175.34 & 31.64 \\
$\mathbf{3}$ & 249.70 & 104.57 & 226.85 & 64.49 & 161.64 & 64.33 \\
$\mathbf{4}$ & 230.62 & 79.20 & 241.82 & 88.88 & 146.40 & 68.26 \\
\hline
\end{tabular}

Figura 2. Latencia en tarea de comprensión morfológica/número de letras a lo largo de presentaciones expresada en msec: $\mathrm{NL}=$ Nivel lector; $\mathrm{BL}=$ Buenos lectores; $\mathrm{DAL}=$ Dificultad lectora .

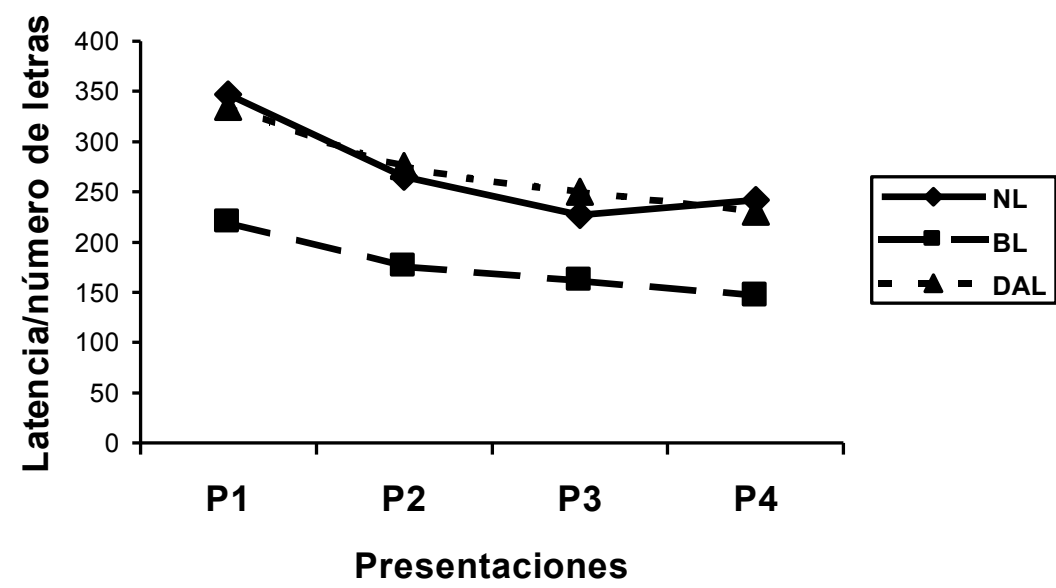




\section{Discusion}

El objetivo de este estudio fue investigar el papel de unidades multiletras, tales como el morfema y la palabra completa, en el reconocimiento de la palabra en niños españoles con dislexia. Los resultados mostraron que había un déficit en el procesamiento léxico en niños con dificultad en lectura comparados con los niños más jóvenes con el mismo nivel lector y no encontramos déficits en el procesamiento morfológico. Sin embargo, cuando analizamos la latencia en la tarea de comprensión morfológica, los resultados mostraron que esta latencia decrecía durante la presentación, independientemente de los grupos, aunque el rendimiento de los buenos lectores, emparejados en edad con los lectores con dificultad, era significativamente mejor que los otros dos grupos.

Con relación al papel de la unidad morfológica (morfema raíz) para acceder al léxico en niños con DAL, nosotros teníamos la hipótesis de que este grupo podría tener más necesidad de utilizar unidades más grandes, como el morfema, en el reconocimiento visual de la palabra porque tienen problemas con la conciencia fonémica. Sin embargo, encontramos que no había diferencias entre los niños españoles con DAL y los otros grupos control en utilizar correspondencias que están basadas en unidades de más alto nivel, tal como el morfema, en una ortografía transparente. Por consiguiente, si no se ha encontrado diferencias entre el grupo con dificultad lectora y los dos grupos control, entonces es improbable que la variable en cuestión (el morfema) juegue algún papel en la explicación de las dificultades lectoras (Bryant y Goswami, 1986). Estos hallazgos son consistentes con investigaciones españolas en las que se ha estudiado el papel de unidades multiletras en el reconocimiento de la palabra. Jiménez, González, Estévez y Hernández-Valle (2000), encontraron que no había diferencias entre los niños con dislexia y los lectores normales en utilizar correspondencias que están basadas en unidades de nivel más alto como el onset y la rima. Asimismo, los hallazgos de Jiménez y Rodrigo (1994) ponen de manifiesto que no hay interacción entre la sílaba y el nivel lector. Esto implica que no hay diferencias entre lectores normales y lectores con dificultad en utilizar la unidad silábica. Sin embargo, algunas ortografías, distintas al español, no son enteramente predecibles sobre las bases de los fonemas ya que no son sistemas transparentes. Esto es así para la notoriamente lengua "irregular", como el inglés y, por extensión, el danés y el francés. Estas lenguas están gobernadas, no sólo por la fonología sino también por la morfología. Por ejemplo, Elbro y Arnbak (1996) llevaron a cabo un estudio sobre el análisis morfológico como estrategia de descodificación de la palabra en un 
grupo de adolescentes daneses con dislexia. Estos autores encontraron que los adolescentes con dislexia, como grupo, mostraban una dependencia de la estructura morfológica de la palabra. Las interacciones indicaron que este grupo estaba significativamente más afectado por la estructura morfológica de la palabra que los lectores normales de los grupos control. El análisis semántico de la palabra fue una ayuda importante para los adolescentes con dislexia, mientras que los lectores normales de los grupos control no utilizan, la estructura semántica para descodificar la palabra. Estos resultados muestran que el reconocimiento del morfema puede ser una estrategia compensatoria para el reconocimiento de la palabra en personas con dislexia.

En resumen, nuestros hallazgos nos permiten contestar a dos cuestiones que habíamos planteado anteriormente. Así, el reconocimiento del morfema contribuye a la lectura de niños con y sin problemas de lectura y esta contribución no es diferente en niños con dificultad lectora y niños lectores normales.

Sin embargo, cuando analizamos la latencia en la tarea de comprensión morfológica, los resultados muestran que esta latencia decrecía durante la presentación, independientemente de los grupos, aunque el rendimiento de los lectores normales emparejados por la edad con los lectores con dificultad, era significativamente mejor que los grupos de niños más jóvenes del mismo nivel lector y que los lectores con dificultad. Estos resultados deben ser interpretados en términos de retraso, no de déficit de los lectores DAL ya que el rendimiento de este grupo es similar al del grupo más joven de igual nivel lector. Posiblemente, este resultado puede ser debido a que ambos grupos tiene menos experiencia con la lengua escrita, y es esta experiencia la que proporciona la oportunidad de adquirir la conciencia morfológica y reconocer la analogía entre las palabras.

Aunque es obvio que con los resultados de la presente investigación no podemos conocer el complejo campo de la morfología, éste es un primer paso para clarificar el papel de la unidad del morfema en el reconocimiento de la palabra en español en niños con dislexia. Será necesario llevar a cabo más estudios con los diferentes tipos de morfología.

En esta investigación también hemos estudiado el papel que juega la palabra completa en el reconocimiento visual de la palabra, en niños españoles con dislexia. Nuestros resultados ponen de manifiesto que los niños con dificultad lectora muestran un déficit en el procesamiento léxico, ya que su rendimiento es menor que el del grupo más joven con el 
mismo nivel lector que los niños DAL. El déficit que hemos encontrado en el procesamiento léxico podría explicarse teniendo en cuenta que nuestra muestra de participantes con dificultad lectora fue seleccionada a partir de la exactitud en la lectura de pseudopalabras, con lo que asumimos que tienen problemas en la decodificación fonológica. Coltheart (1987) especifica que el procesamiento fonológico precede al procesamiento léxico cuando los niños están aprendiendo a leer. Bruck (1993), Share y Stanovich (1995), sugieren que la decodificación fonológica facilita el establecimiento de las representaciones léxicas. También, Alegría (1985) afirma que la construcción del lexicón depende de la ruta fonológica en la lengua española. Además, los lectores normales, en su primera etapa de lectura podrían reconocer palabras frecuentes y no frecuentes utilizando la información del sonido de la palabra. Después, estos lectores estarán en condiciones de utilizar una estrategia léxica para reconocer las palabras familiares (Backman, Bruck, Herbert y Seindenberg, 1984). Sin embargo, los niños con dificultades lectoras tiene problemas en el procesamiento de las relaciones letras-sonido. Esta clase de problemas determina la pobre experiencia con la lectura de los niños con DAL (Stanovich, 1986). Como consecuencia, tienen menos representaciones léxicas que los otros dos grupos porque tienen problemas en el procesamiento fonológico.

En conclusión, los hallazgos de este estudio pueden proporcionar alguna evidencia de que la unidad del morfema no parece ser tan relevante como la unidad palabra completa en el reconocimiento visual de la palabra en español en niños con dislexia. Sin embargo, el procesamiento léxico parece estar más afectado que el morfológico en el grupo de lectores con dificultad.

Los resultados de la presente investigación tienen implicaciones en la práctica educativa. Sugieren que mientras que es necesario entrenar en la estrategia léxica a los niños con dislexia, ya que muestran un déficit en esta habilidad de procesamiento, no parece indicado entrenarles en habilidades morfológicas, ya que, en un sistema ortográfico transparente como el español, el morfema no es imprescindible para acceder al léxico. La experiencia con la lengua escrita en sí misma proporcionará a estos niños la oportunidad de llegar a detectar la analogía morfológica entre las palabras. 


\section{Referencias}

Alegría, J. (1985). Por un enfoque psicolingüístico de la lectura y sus dificultades. Infancia y Aprendizaje, 29, 79-94.

Álvarez, C .J. de Vega, M. \& Carreiras, M. (1998). La sílaba como unidad de activación léxica en la lectura de palabras trisílaba. Psicothema, 10, 371-386.

Álvarez, C.J., Carreiras, M. \& Taft M. (2001). Syllables and Morphemes: Contrasting Frequency Effects in Spanish. Journal of Experimental Psychology: Learning, Memory and Cognition. 27, 2, 545-555.

Backman, J., Bruck, M. Hebert, M. \& Seidenberg, M.S. (1984) Acquisition and use of spelling-sound correspondences in reading. Journal of Experimental Child Psychology, 38 (1), 114-133.

Beech, J. and Awaida, M. (1992). Lexical and no lexical routes: A conmparison between normally achieving and poor readers. Journal of Learning Disabilities, 25, 3, 196-206.

Bruck, M. (1993). Component spelling skills of college sutdents with childhood diagnosis of dyslexia. Learning Disabilities Quarterly, 16, 171-184.

Bryant, P.E. \& Goswami, U. (1986). Strengths and Weaknesses of the Reading. Level Design: A Comment on Backman, Mamen and Ferguson. Psychological Bulletin, 100, 101-103.

Catell, R.B. \& Catell, A. K. S. (1989). Test de Factor “g”. Escala 2. (Cordero, de la Cruz \& Seisdedos, Trans.) Madrid: T.E.A., Ediciones (Original work published 1950).

Cirrin, F.M. (1984). Lexical search speed in children and adults. Journal of Experimental Child Psychology, 37, 158-175.

Coltheart, M. (1987) Lexical access in simple reading task. In G. Underwood (Ed.) Strategies of information processing. London: Academic Press.

Cuetos, F., Rodríguez, B. \& Ruano, E. (1996). Batería de Evaluación de los procesos lectores de los niños de Educación Primaria. (PROLEC) (Assesment test of reading skills for children). Madrid, Spain:T.E.A. 
Daneman, M. \& Carpenter, P.A. (1980). Individual differences in working memory and reading. Journal of Verbal Learning and Verbal Behavior, 19, 450-466.

de Vega, M., Carreiras, M. Gutiérrez Calvo, M. \& Alonso-Quecuty, M. (1990). Lectura y Comprensión: una perspectiva cognitiva. Madrid. Alianza Psicología

Domínguez, A. \& Cuetos, F. (1992) Desarrollo de las habilidades de reconocimiento de palabras en niños con distinta competencia lectora. Cognitiva, 4, 193-208.

Domínguez, A., Cuetos, F. \& de Vega, M. (1993). Efectos diferenciales de la frecuencia silábica: dependencia del tipo de prueba y características de los estímulos. Estudios de Psicología, 50, 5-31.

Domínguez, A., Cuetos, F. \& Seguí, J. (2000). Morphological processing in word recognition: A review with particular reference to Spanish data. Psicológica, 21, 375-401

Domínguez, A., Seguí, J. \& Cuetos, F. (2002). The time-course of inflexional morphological priming. Linguistics 40, 2, 235-259.

Elbro, C. \& Petersen, T. (1993). Udviklingsarbejde om undersogelse af ordblinde elevers laesestrategier. Kobenhavn: Det tvaerkommunale projektsamvirke.

Elbro, C. \& Arnbak, E. (1996). The role of morpheme recognition and morphological awareness in dyslexia. Annals of Dyslexia, 46, 209-240.

Ellis, N. (1981). Visual and name coding in dyslexic children. Psychological Research, 43, 201-218.

Eheri, L.C. and Wilce, L.S. (1983). Development of word identification speed in skilled and less-skilled beginning readers. Journal of Educational Psychology, 75, 3-18.

Frith, U. (1985) Beneath the surface of developmental dyslexia. In K.E. Patterson, J.C. Marshall, \& M. Coltheart (Eds.) Surface Dyslexia. London: Lawrence Erlbaum Associates, Ltd. 
Jiménez, J.E. \& Rodrigo, M. (1994). Is it true that the Differences in Reading Performance between Students with and without LD cannot be explained by IQ ?. Journal of Learning Disabilities, 27, 3, 155-163.

Jiménez, J.E. Guzmán, R. \& Artiles, C. (1997). Efectos de la frecuencia silábica posicional en el reconocimiento visual de palabras y aprendizaje de la lectura. Cognitiva , 1, 3-27.

Jiménez, J.E. (1997). A Reading-Level Design Study of Phonemic Processes Underlying Reading Disabilities in a Transparent Orthography. Reading and Writing: An Interdisciplinary Journal. 9, 23-40.

Jiménez, J.E., Díaz, A., Ortiz, M.R., Rodrigo, M., García, E., Guzmán, R., Hernández-Valle, I., Estévez, A. \& González, G. (2002). SICOLE: A Tutorial Intelligent System in Assessing and Remedial education of Dyslexia in Spanish 2002._Poster session presented in the annual meeting of the International Dyslexia Association. Washington, DC, E.E.U.U.

Jiménez, J.E., Álvarez, C.J., Estévez, A. \& Hernández-Valle, I. (2000). Onset-Rime Units in Visual Word Recognition in Spanish Normal Readers and Children With Reading Disabilities. Learning Disabilities Research \& Practice, 15 (3), 135-141.

Johnston R.S. \& ThompsomG.B. (1989). Is dependence on phonological information in children`s reading a product of instructional approach?. Journal of Experimental Child Psychology, 48, 131-145.

Laxon, V. J., Coltheart, V. \& Keating K. (1988). Children find friendly words friendly too: Words with many orthographic neighbours are easier to read and spell. British Journal of Educational Psychology, 58, 103-119.

Manis, F.R. (1985). Acquisition of word identification in normal and disabled readers. Journal of Educational Psychology, 77, 1, 78-90.

Perfetti, C.A. (1985).Reading ability. New York. Oxford University Press.

Perfetti, C.A. (1986). Continuities in Reading Acquisition. Reading Skill and Reading Disabilities. Remedial and Special Education. 7, 1, 11-21. 
Perfetti, C.A. (1989). There are generalized abilities and one of them is reading. En L.B. Resnick (Ed.). Knowing, learning and instruction: Essays in honor of Robert Glasse. Hillsdale, NJ: Lawrence Eribaum Associates.

Perfetti, C.A. \& Hogaboam, T. (1975). Relationship between single word decoding and reading comprehension Skill. Journal of Educational Psychology, 4, 461-489.

Rack, J.P. Snowling, M.J. \& Olson, R.K. (1992). The nonword reading deficit in developmental dyslexia. A review. Reading Research Quarterly, 27, 20-53.

Rayner, K. (1988). Word recognition cues in children: The relative use of graphemic cues, and grapheme-phoneme correspondence rules. Journal of Educational Psychology, 80, 473-479.

Rodrigo, M. \& Jiménez, J.E. (1999). An analysis of the word naming errors of normal readers and readers disabled children in Spanish. Journal of Research in Reading, 22, 2, 180197.

Rodrigo, M. \& Jiménez, J.E. (2000). IQ or phonological recoding in explaining differences between reading disabled and normal readers in word recognition. Evidence from a naming task. Reading and Writing: An Interdisciplinary Journal, 00: 1-14.

Sánchez-Casas, R. M. (1996). Lexical Access in Visual Word Recognition: the Contribution Word Form. En M. Carreiras, J.E. García-Albea y Sebastián Gallés, N. (eds.), Language Processing in Spanish. Hillsdale, NJ: Erlbaum.

Share, D. C., \& Stanovich, K. E. (1995). Cognitive processes in early reading development: A model of acquisition and individual differences. Issues in Education: Contributions From Educational Psychology, 1, 1-7.

Seymour, P.H.K. (1987). Individual cognitive analysis of competent and impaired reading. British Journal of Special Education, 2, 45-54.

Seymour, P.H.K. \& Porpodas, C. (1980). Lexical and no-lexical processing of spelling in dyslexia. In U. Frith (Ed.), Cognitive Processes in Spelling, 443-473. London: Academic Press. 
Seymour, P.H.K. \& MacGregor, C. J. (1984). Developmental dyslexia: A cognitive experimental analysis of phonological, morphemic and visual impairments. Cognitive Neuropsychology, $1,43-82$.

Siegel, L.S. (1986). Phonolocal deficits in children with reading disabilities. Canadian Journal of Special Education, 2, 1, 45-54.

Siegel, L.S. \& Faux, D. (1989). Acquisition of certain grapheme-phoneme correspondences in normally achieving disabled readers. Reading and Writing : An Interdisciplinary Journal, 1, 37-52.

Siegel, L.S. \& Ryan, E.B. (1988). Development of grammatical-sensitivity, phonological and short term memory skills in normally achieving and subtypes of learning disabled children. Developmental Psychology, 24, 28-37.

Siegel, L.S. \& Ryan, E.B. (1989). The development of working memory in normally achieving and subtypes of learning disabled children. Child Development, 60, 973-980.

Stanovich, K. E. (1986). Matthew effects in reading. Some consequences of individual differences in the acquisition of literacy. Reading Research Quarterly, 21, 4, 360-407.

Stanovich, K.E. (1988). Explaining de differences between the dyslexic and garden variety poor readers: The phonological-core variance-difference model. Journal of Learning Disabilities. 21, 590-604.

Taft, M. \& Forster, K.I. (1976). Lexical storage and retrieval of polymorphemic and polysyllabic words._Journal of Verbal Learning \& Verbal Behavior, 15 (6), 607-620.

Torneus, M. (1987). The importance of metaphonological and metamorphological abilities for different phases of reading development. Paper presented at The Third World Congress of Dyslexia, Brete.

Treiman, R.(1992). The role of intrasyllabic units in learning to read and spell. In PB Gough, LC Ehri, \& R. Treiman (Eds.), Reading acquisition. Hillsdale, NJ: Erlbaum.

Valle, F. (1989). Errores en lectura y escitura. Cognitiva._2 (1), 35-63. 
Working memory in normally achieving and subtypes of learning disabled children. Child Development, 60, 973-980.

Wagner, R.K. \& Torgesen, J.K. (1987). The natural of phonological processing and its causal role in the acquisition of reading skill. Psychological Bulletin,_101, 192-212. 